\title{
Robotics and Assistive Technology to Improve Function in Neuromuscular Diseases
}

\author{
Tariq Rahman ${ }^{1 *}$, Joseph Basante ${ }^{2}$ and Michael Alexander ${ }^{3}$ \\ 'Senior Research Engineer, Department of Biomedical Research, Nemours/ Alfred I duPont Hospital for Children, Department of Mechanical \\ Engineering, University of Delaware \\ 2 Occupational Therapist, Certified Hand Therapist, Nemours/ Alfred I duPont Hospital for Children \\ ${ }_{3}^{3}$ Pediatrics and Physical Medicine and Rehabilitation, Thomas Jefferson University, Philadelphia, Pennsylvania, Chief of Pediatric
}

Rehabilitation

\section{BIOGRAPHICAL NOTES}

Dr. Tariq Rahman (1960) completed his Ph.D. in mechanical Engineering 1990 from Drexel University. He then joined the Alfred I. duPont Hospital for Children as a postdoctoral fellow. Dr. Rahman is currently Senior Research Engineer at the duPont Hospital for Children and Director of the Center for Orthopedics Research and Development (CORD). He also holds the position of Research Professor in the School for Biomedical Engineering at Drexel University and the Department of Mechanical Engineering at the University of Delaware. He has over 65 publications in the area of rehabilitation engineering on issues relating to tremor reduction, robotics, orthotics and orthopedics. He has 5 patents. He organized and chaired the 4th International Conference on Rehabilitation Robotics (ICORR) in 1994, held at the duPont Hospital. He is the founding chair of the steering committee of ICORR (International Conference on Rehabilitation Robotics). He is the inventor of the WREX exoskeleton which is a commercially successful inexpensive device to assist children with arm movement.

Joe Basante (1960) has a Masters degree in Occupational Therapy and a masters degree Exercise Science. He is also currently in the transitional doctoral program in Occupational Therapy at the University of St. Augustine. He currently serves as an Occupational Therapist/ Certified Hand Therapist at Nemours - A. I. duPont Hospital for Children. He has served as a guest lecturer in the Graduate Program for Occupational Therapy at Stockton State College in Pomona NJ. His Specialization areas include treatment and customized splinting for the hand and upper extremity for pediatric populations including hand, wrist, shoulder and elbow pathology. He is a member of The American Occupational Therapy Association, The American Society of Shoulder and Elbow Therapists, The American Society of Hand Therapists and Co-coordinator of the Greater Delaware Hand Special Interest Group. His Publications include: Neuroanatomy/Nerve Injury \& Sensory Reeducation (Chapter Editor.) and Congenital Anomalies/Amputations/prosthetics (Chapter contributor), In Hand \& Upper Extrem-

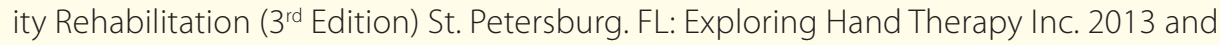
Robotics, Assistive Technology and Occupational Therapy Management to Improve Upper Limb Function in Pediatric Neuromuscular Diseases 2012. Physical Medicine and Rehabilitation clinics. 23(3).

Dr. Michael A Alexander (1947) is a professor of pediatrics and physical medicine rehabilitation at Thomas Jefferson University School of Medicine and is on the Emeritus medical staff at Nemours/Alfred I duPont Hospital for Children. Dr. Alexander 
graduated from the University Of Virginia School Of Medicine in 1972. He was a past president of the American Academy of Cerebral Palsy and Developmental Medicine (1993), and a past president of the Medical Society of Delaware (2000). Dr. Alexander is a medical specialist in Pediatric Physical Medicine and Rehabilitation boarded in Pediatrics and Physical Medicine Rehabilitation. Dr. Alexander was a member of the National Advisory Board on Medical Rehabilitation Research, National Institution of Child Health \& Human Development, and of the NICHD from 1994-1998. He was also on the Board of Trustees of the Commission on Accreditation of Rehabilitation Facilities from 1991-1997. Dr. Alexander also has 12 notable publications over the time span of 1991-2012.

\section{KEYWORDS}

Robotics, rehabilitation, orthotics, occupational therapy.

\section{ABSTRACT}

Therapeutic techniques for people with movement disorders are described along with technology that can be used to mitigate the symptoms. Disorders that affect movement include muscular dystrophy, spinal muscular atrophy, cerebral palsy and others. The article discusses conventional occupation therapy techniques currently used and robotic and orthotic devices that are seen in a research and clinical setting. First the neuromuscular disease presentation is described. Next the current assistive technology techniques and devices are outlined. Finally, robots and orthoses for the upper and lower extremity are described in terms of effectiveness and cost. The robots are divided into assistive and therapeutic sections and they are further treated as passive and active devices.

\section{Introduction}

This review focuses on robotics and other assistive technology used to treat and assist people with neuromuscular conditions such as muscular dystrophy (MD), spinal muscular atrophy (SMA), arthrogryposis multiplex congenita (AMC) and cerebral palsy (CP). These conditions render the extremities of these individuals' weak, uncontrolled, stiff or a combination thereof. People with these conditions resort to using compensatory movements such as ballistic - or swinging - move- ments, using tabletop assist, using both hands, or bending their head forward to get food directly from the plate. These movements, while effective, are inefficient and can be undignified. A parent or caregiver is sometimes asked to help in the feeding process or to assist in other activities of daily living, which can rob people of their independence. Robots, orthoses and other technology can provide the needed assistance to people with disabilities to live an independence and dignified life. Although robots can be mechanical, machine-like and impersonal in appearance and movement they can be of tremendous help also, if matched well with the person and their disability. The last 10-15 years have seen tremendous progress in rehabilitation robotics as technology shrinks and improves and personal machines become more acceptable. This paper describes the medical conditions that require this robotic intervention, the types of assistive technology being used by the therapists, and a review of rehabilitation robots and orthoses.

The use of assistive devices and assistive technology (AT) in environmental modification has been used in OT practice for many years $[1,2,3]$. This could include the use of low-tech devices such as swivel spoons and button hook devices, adaptive equipment such as self-feeding apparatus and overhead slings, and augmentative communication devices.

\section{Neuromuscular Diseases and Presentation}

Neuromuscular conditions present in two distinct motor patterns, a) those that initially present with proximal weakness while sparing fine motor muscles till later in the condition, and b) those that present with distal weakness first and are accompanied with a loss of position, kinesthetic, and proprioceptive awareness. The latter tend to affect fine motor control which makes simple tasks, like buttoning a shirt, arduous. The following neuromuscular conditions are considered in this chapter. People with MD and SMA often use a wheelchair as their primary mode of transportation and as such their activity is confined to the upper limbs. Muscular dystrophy includes Duchenne and Becker muscular dystrophy as the two most common forms of MD. Both are caused by a genetic failure to produce the protein dystrophin. Duchenne muscular dystrophy occurs in approximately one of every 3500 live male births [4]. Becker muscu 
lar dystrophy is much rarer than Duchenne MD. Spinal muscular atrophy is the name given to a group of inherited diseases also characterized by muscle wasting and weakness. It affects one in every 10,000 live births [5]. SMA, like MD, affects the larger muscles closer to the body rather than those at the extremities. As in other forms of neuromuscular disease, decreased movement of an extremity over time leads to contractures and a decrease in the range of motion (ROM).

$\mathrm{CP}$ is the most common orthopedic disorder affecting 2.5 to 5 per 1,000 live births [6]. There are approximately 500,000 people in the United States with cerebral palsy. It is caused by an injury to the immature brain during or shortly after birth. The type of motor involvement is variable and can include: spasticity, athetosis (movement disorder), and mixed (combination of both). Spasticity results from injury to the pyramidal (voluntary) motor system and is defined as an increased stretch reflex that causes muscle stiffness. Spasticity affects about $90 \%$ of the people with CP. Athetosis results from injury to the extrapyramidal motor system causing involuntary movements.

Both upper and lower extremities may be involved in $\mathrm{CP}$, affecting mobility and manipulation. One third of children with CP walk but have significant functional limitations. The upper extremity is frequently involved and decreases the patient's ability to manipulate objects including activities of daily living, writing, drawing, feeding and accessing a computer.

AMC is a consequence of an intrauterine loss of movement which results in joint contractures. Most commonly it is the result of an intrauterine loss of anterior horn cells and in most cases a sparing of sensation and proprioception.

\section{Assistive Technology Currently used in Therapy}

Listed below are some of the more common assistive devices of which many are homemade and use relatively simple household items such as foam blocks and PVC piping. In many cases, the patient and/or family give the OT many good ideas for developing and passing on assistive devices for other families with similar functional challenges!

Some of the more common types of adaptive equipment /assistive devices used are:

- Built up handles on feeding, writing and grooming implements to decrease the amount of force needed to secure and maintain grasp.

- The use of a Universal Cuff with feeding or writing implements in such cases where grip and pinch mechanics are impaired.

- Purchase of specialized feeding equipment such as "Dining with Dignity" in which regular silverware is retrofitted with bendable finger rings to slide over fingers, decreasing the need for a strong grip.

- Attachment of feeding or writing implements onto a splint, whereby gross arm movements are turned into functional tasks.

- The use of "scoop dishes" in which one edge of the dish is built up and angled gently back toward the plate to allow the patient to push the food onto the feeding implement by pushing it up against the "scooped" edge.

- The use of "swivel spoons" in which the distal portion of the spoon is attached to a swivel, allowing the spoon to be angled properly relative to the plate or bowl and be brought to the mouth without spilling if pronation or supination of the forearm is difficult.

- The use of a "Spork" which is a combination of a spoon and a fork to allow the implement to perform multiple functions.

- The use of "Dycem", which is a commercially available "non skid" surface to place under eating containers, much like a place mat, to prevent the plates or bowls from sliding across table while feeding.

- The use of rocker knives to allow a "rocking" motion through use of a curvilinear blade rather than a more difficult linear cutting pattern.

- The use of a long handled reacher to grasp objects outside of the functional range of motion. Reachers with a forearm bracing component are inherently more stable as they shift some of the load to the forearm and not directly to the radial side of the wrist.

- The use of leg lifters to allow arm bracing and trunk control to lift legs into a car or wheelchair.

- The use of "dressing sticks" which have various hooks and or protrusions which enable the patient to grasp and manipulate clothing items while dressing. An adjustable "Swiffer handle" with the cleaning end removed will also allow for easier manipulation of pants and other clothing items when grip is impaired (Marnie King, OT, Wilmington, Delaware, personal communication, 2010). The handle extension can be compacted for easier 
storage and then extended as needed

- The use of Velcro fasteners for shoes, pants and other clothing items. The incorporation of a " $D$ ring" may allow for a child to use crude grip patterns more efficiently by "hooking" the ring along $a$ hand web space to allow for a more gross arm movement to complete dressing tasks when grip and pinch are impaired.

- The use of PVC piping to attach to the end of a faucet handle to act as an "extension" allowing the child to manipulate the control of water flow.

- The attachment of a light dowel to a light switch will enable a child to manipulate the lights from his/her wheelchair.

- Adaptations in seating and positioning using such devices as the "Rifkin Chair" and "Trip Trap Chair", along with appropriate postural stability inserts for proper proximal stability enhancement through biomechanically proper posture.

Many of these items may be combined to suit the patient needs and may be fabricated by the innovative OT after careful analysis of functional deficits and patient goals.

Reducing the effects of gravity are exceptionally important concepts in development of therapy programs for the person with neuromuscular disorders. The reduction of gravity may facilitate function by allowing weakened muscles to contract to a fuller degree and allow for better joint excursion and function. The concept of gravitational grading could be considered to be remedial and/ or compensatory, depending upon the potential for recovery of each particular condition. There are several ways in which this could be accomplished: 1. Foam Blocks or wedges on tabletops can be used to prop arms up and to allow for active assisted or passive shoulder flexion to assist in feeding and or grooming and other tasks requiring hand to mouth movement patterns. Shoulder flexion and/or hand-to-mouth patterning are then achieved by incorporating trunk flexion.

2. The use of an adapted scooterboard with pivoting wheels may be used to facilitate range of motion in friction reduced planes [1]. An excellent variation of this concept is to use a toy car or other toy with 4 wheels to replicate the scooterboard. The car could be attached to the child's extremity with Velcro splint strapping.

3. Positioning the child in a sidelying position with the upper extremity placed on a smooth surface, such as $1 / 4$ particle board propped up on pillows or fabricated into a sturdier bench configuration, will also enhance gravity and friction-reduced motion for shoulder forward flexion. Positioning the child in a supine position with the arm in horizontal abduction and external rotation on the particle board. Shoulder abduction could then be performed. If the particle board is supported at an oblique angle form the front auxiliary line, then shoulder motion could be performed in the scapular plane, which has been stated to be a more functional movement pattern for the shoulder than pure shoulder flexion or pure abduction. A pillow case, towel, powder or lotion could be also be used to further reduce friction (the latter two methods are a bit messy!). If shoulder motion is desired and there is a predominance of flexion at the biceps then a lightweight static elbow splint such as a "pediwrap" or inflatable pneumatic cuff, may be used to keep the elbow in a static position, redirecting motion toward the shoulder. This "offloading" technique may be used for any functional movement pattern for the upper extremity. Depending upon potential for recovery and existing level of strength, a patient may be placed at an appropriate level within the gravity reduction continuum that allows for maximal range of self generated motion with minimal fatigue.

4. PVC pipe stands with a sturdy base can easily be fabricated and used in the bath or shower to prop arms and assist in hand-to-head bathing and grooming (Marnie King, OT, Wilmington, Delaware, personal communication, 2010). In addition, hooks and other types of securing devices can be added for storage of washrags, brushes, etc.

5. Aquatic Therapy is a reasonable therapeutic medium for children who have neuromuscular diseases [7]. A person immersed to the symphysis has effectively offloaded $40 \%$ of his or her bodyweight. When further immersed to the umbilicus, approximately $50 \%$. Xiphoid-level immersion offloads bodyweight by $60 \%$ or more depending on whether the arms are overhead or beside the trunk [8]. Care must be taken however in incremental immersion above chest level secondarily to the effects of hydrostatic pressure and if the patient has impaired respiration. The effects of water buoyancy will enable the child to contract muscles in a gravity-reduced plane and allow for range of motion and play activity.

6. Overhead slings can be fabricated from PVC piping. The slings can be fabricated from neoprene, fabric or soft strapping material. They could be wrist-based or forearm-based depending upon the level of stability desired. The slings are then connected to the PVC frame with the use of therabands, thera tubing, rubber bands or other elastic medium. The child's hands and forearms are then suspended by the slings in order to allow for gravity reduced active-assisted movement.

7. Counter-balanced slings consist of an arm sling attached to a frame via a cable or string using series of pulleys and a counterbalance weight to offload the weight of the upper extremity. The purpose being to allow for functional active-assisted movement in gravity reduced planes. The use of counterbalanced slings allows motion in multiple planes, but they are attached to a large frame which is bulky and often difficult to properly maneuver into biomechanically correct alignment. Additionally, the method of counter 
balance weight delivery may not be as precise as needed for a "zero balance" effect for the upper extremity. The slings may also tend to slip off of the forearm.

8. Balanced Forearm Orthoses (BFO) consists of an adjustable forearm trough attached to a guide post arm which articulates with a swivel. This assembly supports the weight of the forearm and arm against gravity and is used primarily in patients with high-level tetraplegia or severe proximal arm weakness or paralysis. It may be attached to a wheelchair or table; patients may be able to perform tabletop activities; prerequisites for its use include a power source, such as neck or trunk muscles (to shift the trunk center of gravity) or adequate scapular movement [9]. A limitation of the BFO is that it does not allow for shoulder flexion, shoulder abduction or shoulder scapular plane movement.

\section{Rehabilitation Robotics and Orthotics}

Robots were used in the better part of the 20th century in a manufacturing environment, such as automobile assembly. These were repetitive tasks that were labor intensive and required a high degree of accuracy. Towards the latter part of the century the possibility of robots interacting with humans was becoming a possibility. These included advanced prosthetics, motorized feeding devices, and sentry robots [10].These devices were starting to augment or replace mobility and manipulation functions for humans. As WW II was a big driver of improvements in prosthetics technology, the advancement of technology- such as miniaturization of the microprocessor, became a driver for technology development for assistive devices. Another factor was the aging population due to the babyboom generation entering old age. The graying of the population is a world-wide phenomenon particularly in Japan where robotics technology in healthcare was more readily embraced than in many other developed countries.

Robots were first seen in research labs [11] as assistive devices to help people with paralysis of the upper extremity. Later wheelchair-mounted and fixed robots were developed and also commercial industrial robots were adapted for healthcare [12]. These were used for a diverse set of motor disabilities including neuromuscular diseases such as muscular dystrophy and spinal muscle atrophy. Although these devices had a profound effect on people with neuromuscular diseases, widespread commercial success was not seen. A number of factors are thought to contribute to this including weight, human-machine interface, and cost.
In contrast, the rehabilitation robotics field has shifted emphasis in the last 10-15 years towards therapy robots, and the prime beneficiary is the stroke population. This has been driven by the large number of people with stroke; science showing the ability of the brain to adapt through neuroplasticity even in the chronic stage [13], and the need to contain healthcare costs. The last few years have seen more projects looking at cerebral palsy and robots $[14,15,16]$. The following sections are broken up into assistive robots, therapeutic robots and upper extremity orthoses, with an emphasis on devices that currently exist in the market and are used clinically and as consumer products.

\subsection{Assistive Robots and Orthoses}

This section describes the commercially available robots and orthoses including feeders.

A device that has been available for more than 20 years is the iARM (formerly known as the Manus). This is a 7-jointed robot arm that is wheelchair mounted and allows people with neuromuscular conditions to access their environment and perform a subset of tasks their natural arm would. The iARM allows someone to get a drink or feed themselves. It can be controlled by a joystick or keypad. It can be controlled in joint mode, programmed mode or Cartesian mode-which moves the gripper in an xyz configuration. The motors are powered by the wheelchair battery and the iARM sits alongside the wheelchair. The iARM [17] costs $\$ 20,000$. The iARM is used by people with muscular dystrophy, spinal muscular dystrophy, spinal cord injury, cerebral palsy and many other motoric disabilities. There are approximately 400 iARM units in use worldwide.

Upper extremity exoskeletons are used for people who have some residual strength in their arms. This is often seen in people with neuromuscular disorders such as MD, SMA, arthrogryposis, as well as other motor disorders such as ALS and SCI. These exoskeletons are generally passive - meaning that they do not have external power such as motors. They are often attached to wheelchairs and are light weight. One such exoskeleton is the WREX (Wilmington Robotic EXoskeleton). The WREX is a mechanical linkage that can be attached to a wheelchair and is powered by elastic bands $[18,19]$. The device moves alongside the arm and makes anti-gravity movements effortless. This is particularly useful for people with muscular dystro- 
phy and spinal muscular atrophy where weakness in larger proximal muscles is evident while distal muscles are less affected. The WREX allows them to navigate their hand in front of them and perform activities of daily living. The WREX comes in one size and can be adjusted to accommodate different sized individuals and the number of elastic bands can be changed depending on the weight of the individual. The WREX is sold through JAECO [20] and Patterson Medical. The cost is approximately $\$ 2,000$.

The balanced forearm orthosis (BFO) or Ball bearing feeder is a mechanical linkage that hooks on to a wheelchair and allows people to move their arm in the horizontal plane. They are able to move their hand to their mouth by pivoting about a fulcrum at the midpoint of the forearm. There is also a version that allows elevation with an elastic band, however this is rarely used. The BFO was developed in the 1950s and is available through Patterson Medical for approximately $\$ 600$.

The ARMON is available in Europe and is also a wheelchair mounted passive exoskeleton that allows the arm to move against gravity. It uses adjustable springs as the power source and is used for people with neuromuscular conditions such as muscular dystrophy and SMA. The ARMON has a different configuration to the WREX in that it is not a true exoskeleton as it originates from the base of the wheelchair. It allows a large range of gravity free motion. It costs about \$3000 [17].

Another commercially available device is called the DAS (Dynamic Arm Support) [17]. This is also a spring loaded upper extremity orthosis for people with arm weakness. It also attaches to a wheelchair and is available in Europe.

\subsection{Feeders}

Task-specific devices exist for feeding such as the Winsford feeder - sold through Patterson Medical for approximately $\$ 3800$. This is a motorized device intended for people without available arm function. They activate a chin switch, which sends a signal to scoop up food off a mechanized plate and present it to the user. The Neater Eater (Neater Solutions, Buxton, UK) is a table mounted feeding device that comes in two versions. The first is a motorized feeding arm that can be controlled by a user with little arm function and retails for about $\$ 4000$. It is attached to a tabletop and can be controlled by a foot switch. A manual version is also at- tached to a tabletop and is for someone with some arm movement which may be erratic or tremulous. The arm has a built-in damper that filters out unwanted movement.

\subsection{Assistive Orthotics Research}

A cable-driven upper extremity orthosis is being developed at the University of Delaware [21]. The goals are to develop a motorized light weight device that manipulates the arm through cables. A.I. duPont hospital for Children/Nemours is developing a power-assisted UE assistive orthosis to help people with neuromuscular disabilities. A powered device is required if the individual is profoundly weak and retains minimal strength and sensation [22]. Also if they wish to pick up an item, a passive device would not be sufficient. The Scuola Superiore Sant' Anna in Pisa Italy is conducting research in many aspects of rehabilitation robotics for the upper extremity [23].

\subsection{Therapy Robots}

In the last few years a number of therapy robots have become commercially available primarily geared towards the stroke population. The MIT Manus is amongst the earliest of the upper extremity therapy robots. It is a multi-degree of freedom impedance-controlled device. It is capable of moving the user's arm to specific locations or provides partial assist in moving their arm. It has a video monitor and guides the user to locations visually. It has been shown to be as good as manual therapy after a period of exercise [24]. The MIT Manus is sold through Interactive Motion Technologies, Cambridge MA. It is ideal for repetitive delivery of UE therapy in an automated and measurable manner. Another similar device is the Motorika Reo. It is also an UE motorized device that takes a paretic arm through an exercise regimen controlled by a computer screen. The device is available through Motorika Medical Ltd, Caesarea, Israel.

Hocoma AG, Switzerland makes a suite of upper extremity therapy devices. Two of these are the ArmeoSpring and the ArmeoPower. The former is an offshoot of the WREX described earlier and uses springs to counter gravity. It is connected to a computer screen to provide an interactive environment for therapy. Hocoma has sold approximately 250 of these units to date. The newer ArmeoPower is a motorized upper extremity exoskeleton that allows early rehabilitation of motor disorders such as stroke, TBI and other neurological disorders. It is 
based on the ARMIN technology developed at ETH Zurich [25]. The ArmeoPower provides assistance as needed and adapts to the patient's capabilities. There are a host of commercially available lower extremity devices available. These are used for repetitive, body-weight supported movement. Some operate by propelling the legs through the gait cycle. The Locomat by Hocoma AG is perhaps the most prominent among these devices. It is a multidegree-of-freedom robotic device that is treadmillbased. It includes a partial weight-bearing feature [26]. Over 500 Locomat devices are currently in use worldwide.

Motorika offers the ReoAmbulator which integrates body weight supported treadmill therapy and a robotic device similar to the Locomat to promote neural recovery. An interactive display provides feedback and multiple scene modes to increase motivation. Kineassist [27] is an overground mobile base that provides body-weight supported treadmill training in a rehabilitation setting for assistive therapeutic exercises and gait training. It consists of various modes that allow patients to relearn movements and postures by providing a stable and assistive environment to provide overground walking. ZeroG is a new overground body weight supported system that utilizes a roofmounted rail system [28] to provide gait, posture and balance activities. It allows patients to walk on flat terrain or stairs. ZeroG is available through Aretech LLC. Rewalk (Argo medical technologies) is a powered exoskeleton that assists people with walking impairments. It is a motorized device that straps on to the legs and assists in hip and knee flexion. Movement of the legs is initiated by tilting the torso back and forth. Currently the Rewalk is undergoing clinical trials at various therapy centers and hospitals. It requires crutches or a walker to insure balance during gait. Ekso Bionics has developed a device called elegs that offers a powered exoskeleton that propels the legs during gait, similar to the Rewalk. The device is currently undergoing clinical trials in various rehabilitation institutes in the U.S. The projected price of the elegs is approximately $\$ 100,000$.

\section{References and Notes}

[1] Shepherd J, Procter S, Coley IL. 1996. Self-Care and Adaptations for Independent living. In Occupational Therapy for Children 3rd Edition. St. Louis: Mosby. 461-503.

[2] Trombly CA. 2002.Managing deficit of First Level Motor Control Capacities. In: 0c- cupational Therapy for Physical Dysfunction, 5th Edition. Baltimore, MD: Lippincott Williams \& Wilkins. 571-584.

[3] Angelo J, Buning ME. 2002.High Technology Adaptations to Compensate for Disability. In: Occupational Therapy for Physical Dysfunction, 5th Edition. Baltimore, MD: Lippincott Williams \& Wilkins. 389-584.

[4] Bach JR. and Lieberman JS. 1993. "Rehabilitation of the Patient with Disease Affecting the Motor Unit" in Rehabilitation Medicine: Principles and Practice. 2nd Ed. ed Joel A. DeLisa. J.B. Lippincott Co.

[5] Rahman T. Stroud S, Ramanathan R, Alexander M, Seliktar R. and Harwin W. 1996 "Task Priorities and Design for an Arm Orthosis" Technology and Disability Journal. Vol. 5(2), pp 197-203.

[6] Miller F. 2005. Cerebral Palsy. New York, New York, United States of America: Springer Science + Business Media, Inc.

[7] McDonald CM. 2010. Neuromuscular Diseases. In: Alexander MA, Matthews DJ, editors. Pediatric Rehabilitation- Principles and Practice, 4th edition. New York: Demos Medical. pp. 277-335.

[8] Becker BE. 2009. Aquatic therapy: scientific foundations and clinical rehabilitation applications. PM R;1:859-72.

[9] Lansang R. 2012. Upper Limb Support Devices. In: Medscape Reference: Drugs, Diseases and Procedures. Available at: http://emedicine.medscape.com/ article/314774-overview. Accessed April 17

[10] Thring MW. 1983. Robots and Telechirs: Manipulators with Memory, Remote Manipulators, Machine Limbs for the Handicapped (Ellis Horwood series in engineering science). Chichester, UK: Halsted Pr;

[11] LeBlanc M, Leifer L.1982. Environmental Control and Robotic Manipulation Aids. Engineering in Medicine and Biology Magazine; 1:16-22.

[12] Burgar CG, Lum PS, Shor PC. et al. 2000. Development of robots for rehabilitation therapy: The Palo Alto VA/Stanford experience. J Rehabil Res Dev; 37:863-73.

[13] Krebs HI, Hogan N, Aisen ML, et al. 1998. Robot-aided Neurorehabilitation. IEEE Trans Rehabil Eng; 6:75-87.

[14] Fasoli SE, Fragala-Pinkham M, Hughes R, et al. 2008. Upper limb robotic therapy for children with hemiplegia. Am J Phys Med Rehabil; 87:929-36.

[15] Fasoli SE, Fragala-Pinkham M, Hughes R, et al. 2008. Robotic therapy and botulinum toxin type A: A novel intervention approach for cerebral palsy. Am J Phys Med Rehabil;87; 1022-5.

[16] Cioi D, Kale A, Burdea G, et al. 2011. Ankle Control and Strength Training for Children with Cerebral Palsy using the Rutgers Ankle CP: A case study. 2011 IEEE International Conference on Rehabilitation Robotics, Zurich Science City, Switzerland, June 29 July 1.

[17] Assistive Innovations. Assistive Innovations product listing. In: Assistive Innovations index page. Available at: www.assistive-innovations.com. Accessed April 17, 2012.

[18] Rahman T, Sample W, Seliktar R, et al. 2007. Design and testing of a functional arm orthosis in patients with neuromuscular diseases.IEEE Trans Neural Syst Rehabil Eng; 15.244-51.

[19] Haumont T, Rahman T, Sample W, et al. 2011. Wilmington robotic exoskeleton: a novel device to maintain arm improvement in muscular disease. J Pediatr Orthop; 31:e44-9.

[20] Jaeco Orthopedic. WREX: Wilmington Robotic Exoskeleton Arm. In: Jaeco Orthopedic product listing. Available at: http://jaecoorthopedic.com/products/products/ WREX\%3A-Wilmington-Robotic-EXoskeleton-Arm.html. Accessed April 17, 2012. 
Acta Mechanica Slovaca

Journal published by Faculty of Mechanical Engineering - Technical University of Košice

[21] Mao Y, Agrawal S. 2011. A Cable Driven Upper Arm Exoskeleton for Upper Extremity Rehabilitation. ICRA IEEE International Conference:4163-68.

[22] Ragonesi D, Agrawal S, Sample W, et al. 2011. Series elastic actuator control of a powered exoskeleton. Conf Proc IEEE Eng Med Biol Soc:3515-8.

[23] Cavallo F, Aquilano M, Bonaccorsi M, et al. 2011. Multidisciplinary approach for developing a new robotic system for domiciliary assistance to elderly people. Conf Proc IEEE Eng Med Biol Soc:5327-30.

[24] Lo AC, Guarino PD, Richards LG, et al. 2010. Robot-assisted therapy for long-term upper-limb impairment after stroke. N Engl J Med;362;1772-83.

[25] Brokaw EB, Murray T, Nef T, et al. 2011. Retraining of interjoint arm coordination after stroke using robot-assisted time-independent functional training. J Rehabil Res Dev;48:299-316.

[26] Westlake KP, Patten C. 2009. Pilot study of Lokomat versus manual-assisted treadmill training for locomotor recovery post-stroke. J Neuroeng Rehabil ;6:18.

[27] Kinea Design. Portfolio: KineAssist Walking \& Balance retraining. In: kinea Design product portfolio. Available at: http://www.kineadesign.com/portfolio/kineassist/. Accessed April 17, 2012.

[28] Aretech, LLC. ZeroG Overview. In: Aretech, LLC Home page. Available at: http://www. aretechllc.com/overview.html. Accesed April 17, 2012. 